\title{
High sensitivity microsensor assisted by Fano resonance in InSb based terahertz plasmonic waveguide
}

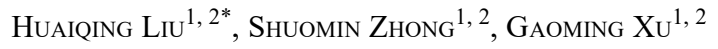 \\ ${ }^{1}$ Faculty of Electrical Engineering and Computer Science, \\ Ningbo University, Ningbo 315211, China \\ ${ }^{2}$ Laboratory of Infrared Material and Devices, Advanced Technology Research Institute, \\ Ningbo University, Ningbo 315211, China \\ *Corresponding author: liuhuaiqing@nbu.edu.cn
}

\begin{abstract}
We propose a compact terahertz plasmonic structure comprising an InSb-dielectric-InSb waveguide side-coupled with two stub resonators. Due to the coherent interference of the splitting discrete and quasi-continuum modes, the reflection spectrum possesses a sharp asymmetric Fano resonance dip, which stems from the phase difference between the two stub resonators. Owing to the permittivity temperature dependent property of InSb, the Fano resonance dip can be actively controlled by tuning temperature. The physical features contribute to a highly efficient plasmonic sensor for both refractive index and temperature sensing. The microsensor yields a sensitivity of $\sim 2.9 \mathrm{THz} / \mathrm{RIU}$ and $1.8 \times 10^{-3} \mathrm{THz} /{ }^{\circ} \mathrm{C}$. This multiparameter high sensitivity microsensor may find important applications in medical sensing, biosensing and on-chip sensing working in terahertz region.
\end{abstract}

Keywords: plasmonic, microsensor,terahertz,Fano resonance.

\section{Introduction}

Surface plasmon polaritons (SPPs) have shown the considerable potential to control light at the subwavelength scale due to their ability of overcoming the diffraction limit [1]. Recently, huge kinds of highly integrated optical devices have been investigated or demonstrated assisted by plasmonic nanostructures, such as perfect absorbers [ $[\underline{2}, \underline{3}]$, fil-

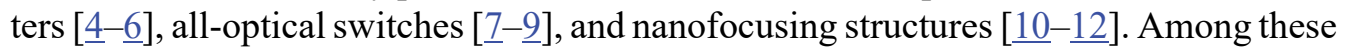
devices, plasmonic sensors for refractive index sensing have been explored by means of the classical analogue of electromagnetically induced transparency (EIT) [13-15] or perfect absorption resonance [16-18]. However, the sensitivity of the sensors based on traditional surface plasmon resonance (SPR) can hardly get too high attribute to the symmetry property of the traditional SPR. The resonance, discovered by Ugo Fano, originates from the quantum-mechanical interference between a discrete excited state of an atom and a continuum sharing the same energy level [19]. Different from the Lorentz 
resonance, the Fano resonance possesses a distinctly asymmetric line profile, which made it superior to Lorentz resonance in plasmonic sensing applications with respect to the sensitivity of the sensors [리]. Thus, designing plasmonic nanosensors based on Fano resonance mechanism seems intriguing and promising.

On the other hand, terahertz $(\mathrm{THz})$ technology has drawn an extensive attention in relation to various application fields such as biological science, medical imaging, security and space science [21-24]. So, there is an urgent need to find a material that can support THz SPP wave. Graphene seems a good candidate for supporting THz SPP wave, and graphene has been introduced to construct broadband terahertz absorbers due to its exceptional properties, such as optical transparency, flexibility, and tenability [25]. However, the structure is demanding in terms of cost and complexity. Another alternative promising material for supporting $\mathrm{THz} \mathrm{SPP}$ wave is a moderately doped semiconductor, which can be readily fabricated using conventional micro-fabrication techniques. Some semiconductors have a permittivity at $\mathrm{THz}$ range close to that of metal at optical range. InSb, whose permittivity can be modified by varying temperature, has been proposed for designing THz plasmonic devices [26-29], so plasmonic $\mathrm{THz}$ sensor based on $\mathrm{InSb}$ material for both refractive index and temperature sensing seems promising and interesting.

In this paper, we propose and investigate a plasmonic microsensor based on Fano resonance in an InSb-dielectric-InSb (IDI) waveguide resonator system, which consists of an IDI waveguide side-coupled with a pair of stub resonators. The theoretical model shows that the Fano resonance lineshape originates from the splitting resonance modes with different coupling rates and resonance frequencies. The Fano resonance dip strongly depends on the separation between the two stub resonators. Owning to the permittivity temperature dependent property of $\mathrm{InSb}$, the proposed microsensor can be utilized for both refractive index and temperature sensing applications. It is superior to the previous work about a multiparameter sensor assisted by complicated frequency selective surface couplers in terms of fabrication procedure [30]. This simple structure multiparameter high sensitivity microsensor may find important applications in medical sensing, biosensing and on-chip sensing operating at terahertz frequency.

\section{Structure design and optical property of InSb material}

The side view of the two-dimensional (2D) schematic diagram of the plasmonic configuration is shown in Fig. 1, which consists of a bus waveguide side coupled with two microcavities with length $l$, width $w$, and a cavity-cavity separation $d$. Since the width of the slit waveguide is much smaller than the incident wavelength, only a single propagation mode $\mathrm{TM}_{0}$ can exist in the structure. Here, the dispersion relation of the fundamental SPP mode in the InSb slot waveguide is determined by [27]

$$
\tanh \sqrt{n_{\mathrm{eff}}^{2}-\frac{\varepsilon_{\mathrm{d}} k_{0} w}{2}}=\frac{-\varepsilon_{\mathrm{d}} \sqrt{n_{\mathrm{eff}}^{2}-\varepsilon_{\mathrm{InSb}}(\omega)}}{\varepsilon_{\mathrm{InSb}}(\omega) \sqrt{n_{\mathrm{eff}}^{2}-\varepsilon_{\mathrm{d}}}}
$$




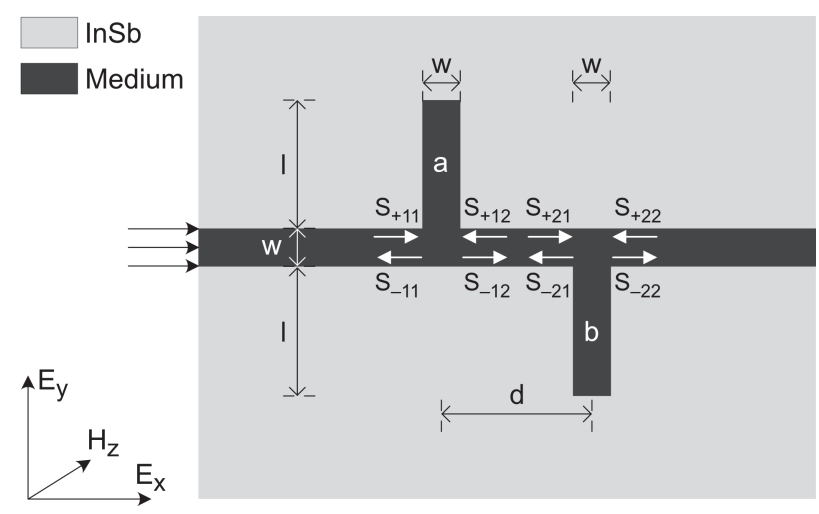

Fig. 1. Schematic diagram of proposed plasmonic system with an InSb slot waveguide side-coupled with a pair of stub resonators; the incident wave is illuminated from the left side.

where $n_{\text {eff }}$ is the effective refractive index of the SPP wave in the waveguide and $k_{0}=2 \pi / \lambda_{0}$ is the free-space wave vector and $\beta=k_{0} n_{\text {eff }}, \lambda_{0}$ is the wavelength of the incident source. The insulator in the dielectric core has permittivity $\varepsilon_{\mathrm{d}}$, and $w$ and $\omega$ denote the width of the two cavities and the angular frequency of the incident wave, respectively.

From 0.1 to $2.2 \mathrm{THz}$, the permittivity of InSb can be approximately given by the simple Drude model approximation [31]

$$
\varepsilon(\omega)=\varepsilon_{\infty}-\frac{\omega_{\mathrm{p}}^{2}}{\omega^{2}+i \gamma \omega}
$$

where $\varepsilon_{\infty}$ represents the high-frequency permittivity, $\omega$ is angular frequency, and $\gamma$ is the damping constant. The plasma frequency $\omega_{\mathrm{p}}=\left(N e^{2} / \varepsilon_{0} m^{*}\right)^{1 / 2}$ depends on the intrinsic carrier density $N$, the electronic charge $e$, the vacuum permittivity $\varepsilon_{0}$, and the effective mass $m^{*}$ of free carriers. One of the great differences between InSb and Ag is that the plasma frequency $\omega_{\mathrm{p}}$ of InSb increases exponentially with increasing tempera-

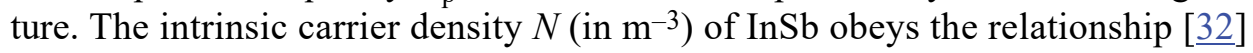

$$
N=5.76 \times 10^{20} T^{1.5} \exp \left(-\frac{0.26}{2 k_{\mathrm{B}} T}\right)
$$

where $k_{\mathrm{B}}$ is the Boltzmann constant and $T$ is the temperature in Kelvins. It should be noted that the damping constant $\gamma$ of InSb is proportional to the electron mobility $\mu$ as $\gamma=\mathrm{em}^{*} / \mu$, which in turn depends on temperature. Thus, while changing the temperature, $\gamma$ will change as well and then it will influence the absorption property of InSb. However, when the temperature ranges from 160 to $350 \mathrm{~K}$ within the frequency regime from 0.1 to $2.2 \mathrm{THz}$, the electron mobility $\mu$ changes slightly. Consequently, the damping constant $\gamma$ can be seen as a constant, which is consistent with the experimental report [33]. For InSb $\varepsilon_{\infty}=15.6, m^{*}=0.015 m_{\mathrm{e}}\left(m_{\mathrm{e}}\right.$ is the mass of electron), and $\gamma=0.1 \pi \mathrm{THz}$ [34]. 


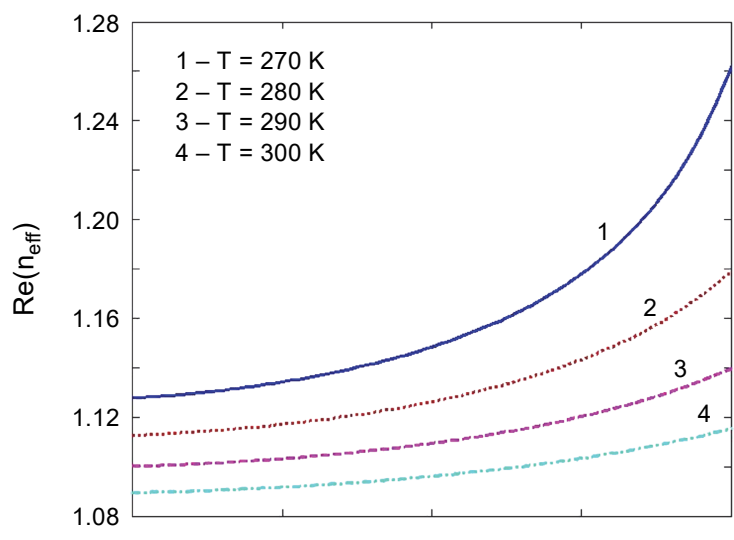

a

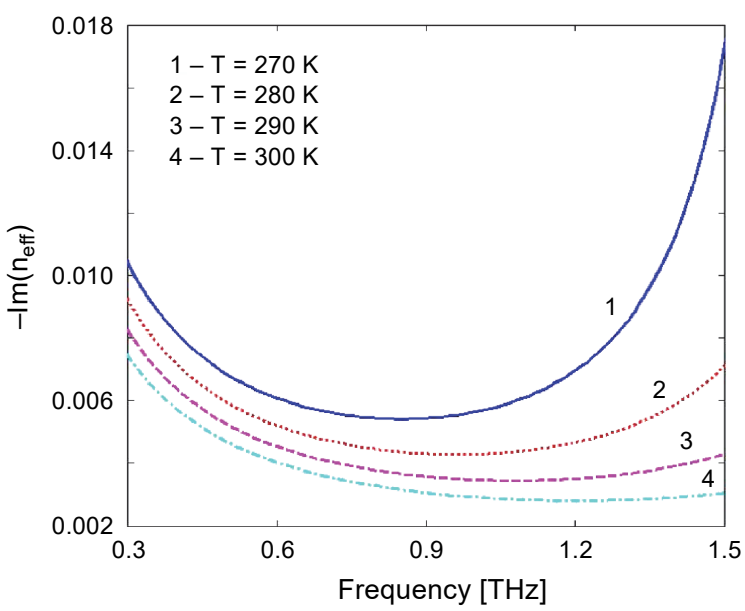

b

Fig. 2. Real part of the effective refractive index of InSb slot waveguide as a function of frequency for different temperatures (a). Imaginary part of the effective index of InSb slot waveguide versus frequency for different temperatures (b). The width of the slit is set as $50 \mu \mathrm{m}$ in both figures.

Figures $2 \mathbf{a}$ and $2 \mathbf{b}$ show the dependence of the real part and imaginary part, respectively, of the effective refractive index of InSb slot as a function of frequency at different temperatures with the width of the slot waveguide and the stub resonators kept as $w=50 \mu \mathrm{m}$. As shown in Fig. 2, we can see that the effective refractive index of $\mathrm{InSb}$ is affected by the variation of the temperature at $\mathrm{THz}$ region. Therefore, tunable plasmonic $\mathrm{THz}$ devices based on InSb material by tuning temperature seem interesting and promising. In this work, the simulations were performed by Comsol Multiphysics based on the finite element method (FEM).

\section{Fano resonance in the proposed THz plasmonic waveguide system}

In the plasmonic waveguide-coupled cavity, the localized resonance can be excited when the frequency of the incident wave approaches the intrinsic resonance frequency. 
The spectrum features of this plasmonic waveguide system can be investigated by the temporal coupled-mode theory [35]. To simplify the analytical model, the propagation and coupling losses are not considered, and the medium is assumed as air $\left(n_{\text {air }}=1\right)$ at first. The schematic diagram of the proposed plasmonic structure for achieving Fano resonance is shown in Fig. 1. For the harmonic time dependence of $\exp (-j w t)$, the time evolution of amplitudes $a$ and $b$ of the above and the below cavity can be respectively described as

$$
\begin{aligned}
& \frac{\mathrm{d} a}{\mathrm{~d} t}=\left(-j \omega_{0}-\kappa_{0}-\kappa_{1}\right) a+\exp (j \theta) \sqrt{\kappa_{1}} S_{+11}+\exp (j \theta) \sqrt{\kappa_{1}} S_{+12} \\
& \frac{\mathrm{d} b}{\mathrm{~d} t}=\left(-j \omega_{0}-\kappa_{0}-\kappa_{1}\right) a+\exp (j \theta) \sqrt{\kappa_{1}} S_{+21}+\exp (j \theta) \sqrt{\kappa_{1}} S_{+22}
\end{aligned}
$$

Here $\omega_{0}$ represents the resonance frequency of cavities; $\kappa_{0}$ is the decay rate of the field due to internal loss in cavities, and $\kappa_{1}$ stands for the decay rate of cavities due to the power escape through the waveguide. These physical parameters are set to be constant with fixed geometrical properties. $\theta$ is the phase of the coupling coefficient. The outgoing waves of cavities can be expressed as

$$
\begin{aligned}
& S_{-12}=S_{+11}-\exp (-j \theta) \sqrt{\kappa_{1}} a \\
& S_{-11}=S_{+12}-\exp (-j \theta) \sqrt{\kappa_{1}} a \\
& S_{-21}=S_{+22}-\exp (-j \theta) \sqrt{\kappa_{1}} b \\
& S_{-22}=S_{+21}-\exp (-j \theta) \sqrt{\kappa_{1}} b
\end{aligned}
$$

The propagation SPP wave in this waveguide should satisfy the relationship: $S_{+12}=S_{-21} \exp (j \varphi)$ and $S_{+21}=S_{-12} \exp (j \varphi)$. Here, $\varphi=2 \pi \operatorname{Re}\left(n_{\text {eff }}\right) d / \lambda$ is the phase difference between the two stub resonators, $\lambda$ is the wavelength of the incident wave, and $n_{\text {eff }}$ is the effective refractive index of the supported THz SPP mode, which can be obtained from dispersion equations. If the incident $\mathrm{THz}$ wave is illuminated only from the left port $\left(S_{+22}=0\right)$, the line shape of the reflection spectrum is expressed as

$$
R=|r|^{2}=\left|\frac{S_{-11}}{S_{+11}}\right|^{2}
$$

The reflection coefficient $r$ can be written as

$$
\begin{aligned}
& r=\exp (j \varphi)\left(r_{1}-r_{2}\right) \\
& r_{1}=\frac{\kappa_{1}(1-\cos \varphi)}{j\left(\Delta \omega-\kappa_{1} \sin \varphi\right)+\kappa_{0}+\kappa_{1}(1-\cos \varphi)} \\
& r_{2}=\frac{\kappa_{1}(1+\cos \varphi)}{j\left(\Delta \omega+\kappa_{1} \sin \varphi\right)+\kappa_{0}+\kappa_{1}(1+\cos \varphi)}
\end{aligned}
$$


Here $\Delta \omega=\omega_{0}-\omega$. The reflection coefficient is written as a form of the interference between two new resonance modes with the frequencies of $\omega_{0} \pm \kappa_{1} \sin \varphi$, coupling decay rates of $\kappa_{1}(1 \pm \cos \varphi)$, and identical intrinsic decay rates. The relationship between the total reflection $R$ and $r_{1}$ and $r_{2}$ can be expressed as

$$
R=\left|r_{1}\right|^{2}+\left|r_{2}\right|^{2}-2\left|r_{1}\right|\left|r_{2}\right| \cos \left(\theta_{2}-\theta_{1}\right)
$$

In the above equation, the first two terms stand for the sum of reflection of two stub cavities, and the last term represents the interference between them. It is obvious that the total reflection $R$ is determined by the phase difference between the two resonant modes of the two stub cavities. While the phase difference of the two resonant modes is determined by the distance between the two stub cavities, so the distance between the two stubs $d$ plays an important role in the response of the whole $\mathrm{THz}$ plasmonic system. At first, in order to more clearly understand the influence of the distance between the two stubs on the reflection spectra of this THz plasmonic system, the geometry parameters of this $\mathrm{THz}$ plasmonic waveguide are set to be $w=50 \mu \mathrm{m}, l=300 \mu \mathrm{m}$. The medium filled in the micro slit and the two stubs is chosen as air and the temperature is kept at $280 \mathrm{~K}$. Under these conditions, the simulated results of the total reflection $R$ for various distances between the two stubs are shown in Fig. 3a. As shown in Fig. 3a, one can see that the reflection spectra are asymmetric and there is an obvious dip which exists in those reflection spectra except for $d=320 \mu \mathrm{m}$, and the dip sometimes locates at the left side and sometimes at the right side, which originates from different coupled-resonator effect. Meanwhile, we can see that the Fano resonance dip disappears in the reflection spectra when $d$ is $320 \mu \mathrm{m}$. According to the dispersion relation of this structure, $n_{\text {eff }}$ of the waveguide is about 1.13 at $1.07 \mathrm{THz}$. Thus, the phase term $\varphi$ in Eqs. (7a)-(7c) is approximately equal to $\pi$ at $1.07 \mathrm{THz}$ when $d=320 \mu \mathrm{m}$. The resonant mode $r_{2}$ vanishes, so the total reflection can be expressed as $R=\left|r_{1}\right|^{2}$ and the Fano resonance dip disappears [ $\underline{36}$ ]. It should be noted that the Fano resonance
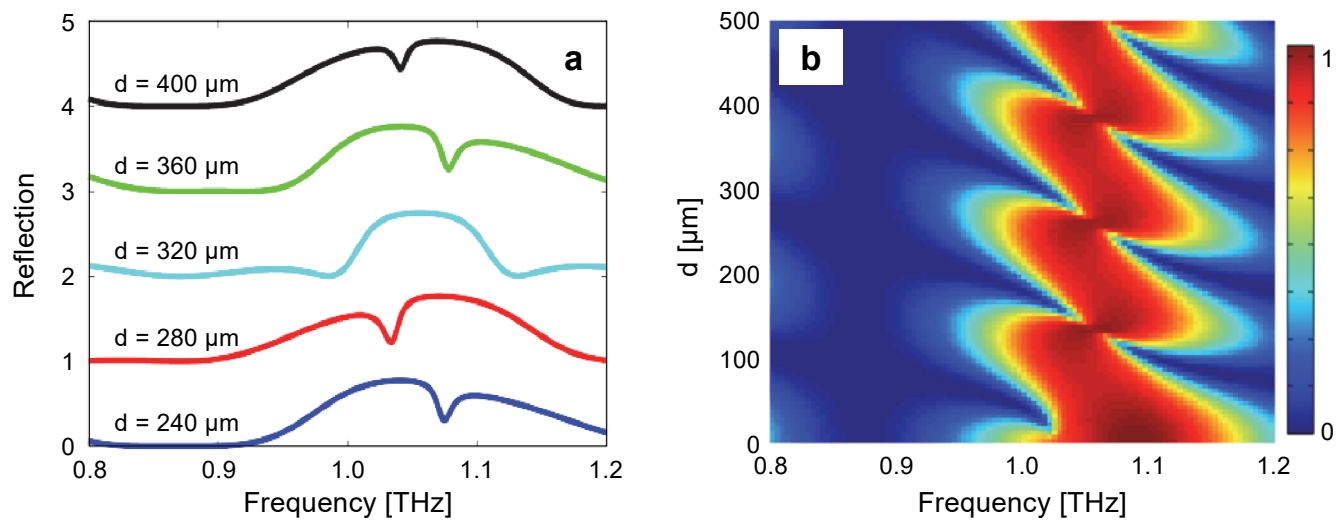

Fig. 3. Total reflection spectra of our THz plasmonic system for various distances between the two stubs (a). The reflection spectra as a function of the distance between the two stub and the frequency of the incident source (b). 
dip possesses a redshift around $d=320 \mu \mathrm{m}$ (i.e., $\varphi=\pi$ ), and this is attributed to the variation of the discrete-state resonant mode $r_{2}$, whose frequency $\omega=\omega_{0}+\kappa_{1} \sin \varphi$ decreases when increasing $d$ around the phase $\varphi=\pi$. The simulation results agree well with the theoretical results in Fig. $3 \mathbf{b}$. Seeing from Fig. $3 \mathbf{b}$, it is found that the reflection spectra evolves periodically with $d$, which is due to the fact that the phase difference between the two stub resonators varies periodically with $d$. This Fano resonance reflection spectrum may find potential applications in micro-sensing and switching in THz system.

In order to get a better understanding of the difference between the Fano-resonance induced spectra and the traditional resonance induced Lorentz-like spectra, we plotted the reflection spectra of our $\mathrm{THz}$ plasmonic system with double stubs and single stub, as shown in Fig. 4a. Clearly, it is observed that the reflection spectra (blue solid line in Fig. 4a) in the two coupling resonators become sharp and asymmetric, which is quite different from that in the single-resonator case (red dashed line in Fig. 4a). For the double stubs system, the reflection spectra of the SPPs varies sharply from the resonance peak to the dip with only a small frequency shift of about $\Delta f=0.038 \mathrm{THz}$ (blue solid line in Fig. $4 \mathbf{a})$, which is obviously smaller than that $(\Delta f=0.21 \mathrm{THz})$ in the single-stub
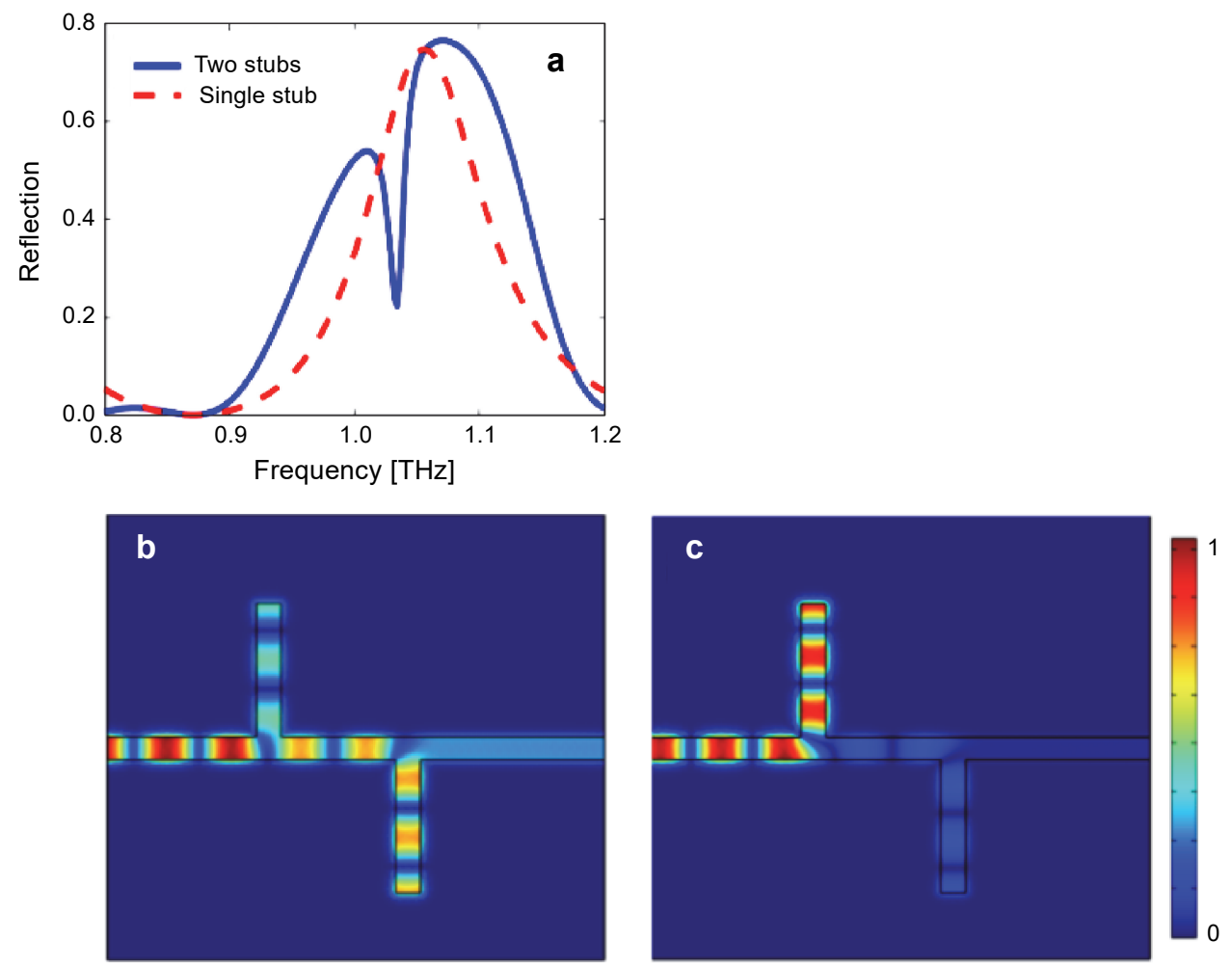

Fig. 4. Total reflection spectra of our THz plasmonic system for both double stubs (blue solid line) and single stub (red dashed line) (a). The normalized magnetic field distributions at the Fano-like reflection $\operatorname{dip}(\mathbf{b})$ and peak (c). The geometric parameters are the same as for Fig. 3, the temperature is kept at $280 \mathrm{~K}$. 
case. In order to get a deep insight of the Fano-resonance effect, the normalized magnetic field distributions corresponding to the reflection dip $(1.034 \mathrm{THz})$ and the reflection peak $(1.072 \mathrm{THz})$ are displayed in Figs. $4 \mathbf{b}$ and $4 \mathbf{c}$, respectively. Obviously, at $f=1.034 \mathrm{THz}$, strong field distributions are observed in the Fabry-Pérot (FP) resonator, which is attributed to the high reflectivity of the stub resonators. This serves as a direct evidence that a strong trapped resonance occurs in the FP resonator, resulting in a narrow reflection dip at $f=1.034 \mathrm{THz}$, while at $f=1.072 \mathrm{THz}$ the SPPs are blocked by the stub resonators, and the output of the InSb-dielectric-InSb waveguide is dark, as shown in Fig. 4c. Thus, the sharp and asymmetric spectra, usually termed as Fano resonances, result from the coupling of a narrow discrete resonance (strong trapped resonance in the FP resonator) and a broad spectrum (weak resonance in the stub resonators). It is shown that the frequency shift of two stub systems required for a completely on/off variation is significantly reduced in the Fano resonance reflection spectra compared to the traditional resonance reflection spectra, implying a high sensitivity to the index variations of nearby or surrounding medium, which makes it a good candidate for THz micro-sensing technology.

\section{Sensing applications based on THz Fano resonances}

Since temperature can affect the permittivity of InSb, so we kept temperature as $280 \mathrm{~K}$ in order to study the complex refractive index sensing properties of the Fano resonance in our THz plasmonic system. We arrange our simulation steps as follows. Firstly, the real part of the refractive index ranges from 1 to 1.04 with a step of 0.02 , and the corresponding reflection spectra are plotted in Fig. 5.

Seeing from Fig. 5, it shows that the resonance wavelength has a redshift when increasing the refractive index of the medium filled in the cavities. This phenomenon results from altering the resonance frequency of this $\mathrm{THz}$ plasmonic system when chang-

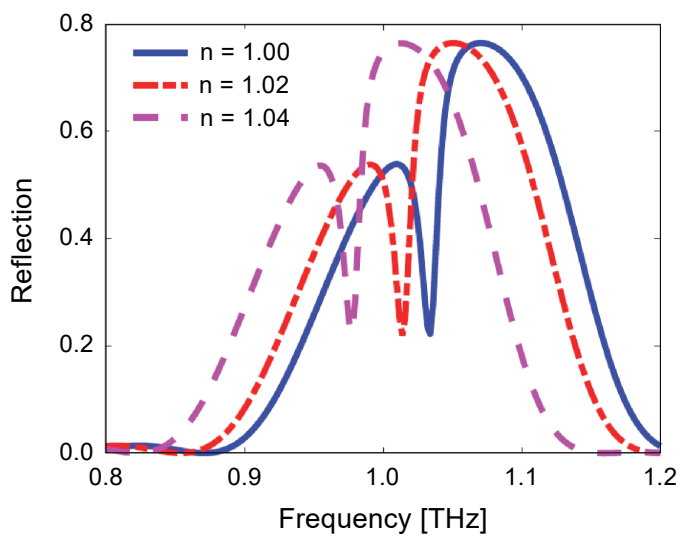

Fig. 5. Total reflection spectra of our THz plasmonic system for various distances between the two stubs. 
ing the refractive index of the medium filled in the cavities. According to the analytical model, the resonance frequency of the splitting mode $r_{2}$ increases with the refractive index. Thus, the Fano resonance dip exhibits a sensitive shift while changing the refractive index of the dielectric. This feature offers an excellent approach for the applications toward microscale sensing. The sharp asymmetric Fano line shape enhances the sensitivity of sensors. Here, assisted by the Fano line shape reflection spectrum, the sensitivity of this microsensor can be as high as $2.9 \mathrm{THz} / \mathrm{RIU}$, which is far more sensitive to refractive index changes in comparison with the sensor based on the traditional Lorentz-like resonance spectra, as the dashed line in Fig. 4a. So this structure can be a good candidate for micro-sensing applications.

On the other hand, due to the fact that the permittivity of InSb is sensitive to temperature, this THz plasmonic system can also act as a temperature sensor without the need of introducing another medium. Here, in order to investigate our structure's sensitivity to temperature, the medium filled in the cavities and the slit is kept as air, and the geometric parameters are the same as in Fig. 3, and then we vary the temperature from 280 to $300 \mathrm{~K}$ with a step of $10 \mathrm{~K}$, The simulated corresponding Fano-like reflection spectra are plotted in Fig. 6 . We can see that the resonance dip of the reflection spectra has a blueshift when increasing temperature, and this phenomenon originates from the fact that the effective refractive index of the SPP mode in our structure gets smaller when increasing temperature according to Fig. 2a, leading to a higher resonance frequency according to the Fabry-Pérot resonance theory. Moreover, the sensitivity of this structure for temperature can be as high as $1.8 \times 10^{-3} \mathrm{THz} /{ }^{\circ} \mathrm{C}$, so this structure can also act as a temperature sensor. Additionally, it should be noted that the resonance dip of the reflection spectra gets lower and lower and the peak value gets higher and higher, which results from the fact that the loss of our structure gets smaller when increasing temperature according to Fig. $2 \mathbf{b}$, leading to lower energy dissipation

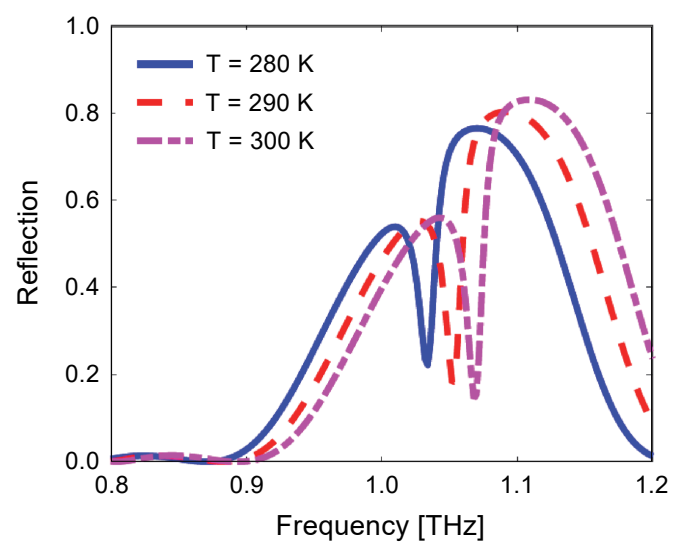

Fig. 6. Total reflection spectra of our THz plasmonic system for various temperatures of the surroundings. The medium filled in the cavities is assumed as air, the geometric parameters are the same as for Fig. 3 . 
in the cavities at the Fano-resonance frequency and more energy transmits out of our structure. Thus, this compact structure can find applications in micro-sensing technology for both refractive index and temperature sensing.

For practical applications, the fabrication process of our structure should also be taken into account, the InSb-air-InSb waveguide can be obtained by suing electron beam lithography (EBL) technology or focused ion beam (FIB) lithography on InSb rectangular waveguide, and then we can put it on the molten state dielectric to get the InSb -dielectric-InSb waveguide by magnetron sputtering technology [37]. Then the THz plasmonic multi-parameters microsensor can be achieved after the air-dry procedure.

\section{Conclusion}

In summary, we have proposed an InSb-dielectric-InSb $\mathrm{THz}$ plasmonic waveguide consists of a slot waveguide side coupled with a pair of stub cavities. Owning to the coherent interference of the splitting discrete and quasi-continuum modes, the reflection spectrum possesses a sharp asymmetric Fano resonance dip. Since the interference between the two stub resonators is strongly dependent on the distance between the two stub resonators, so the shape of the Fano-like reflection spectra is determined by the distance between the two stub resonators. The physical features contribute to a highly efficient plasmonic sensor for both refractive index and temperature sensing. The microsensor yields a sensitivity of $\sim 2.9 \mathrm{THz} / \mathrm{RIU}$ and $1.8 \times 10^{-3} \mathrm{THz} /{ }^{\circ} \mathrm{C}$. This multiparameter high sensitivity microsensor may find important applications in medical sensing, biosensing and on-chip sensing operating at terahertz frequency.

Acknowledgements - This work was supported by the Natural Science Foundations of China (Grant Nos. 61631012 and 61271085). This work was partially sponsored by K.C. Wong Magna Fund in Ningbo University.

\section{References}

[1] Bozhevolnyi S.I., Volkov V.S., Devaux E., Laluet J.-Y., Ebbesen T.W., Channel plasmon subwavelength waveguide components including interferometers and ring resonators, Nature 440, 2006, pp. 508-511, DOI: 10.1038/nature04594.

[2] Li Y., An B., Jiang S., Gao J., Chen Y., Pan S., Plasmonic induced triple-band absorber for sensor application, Optics Express 23(13), 2015, pp. 17607-17612, DOI: 10.1364/OE.23.017607.

[3] Liu N., Mesch M., Weiss T., Hentschel M., Giessen H., Infrared perfect absorber and its application as plasmonic sensor, Nano Letters 10(7), 2010, pp. 2342-2348, DOI: 10.1021/n19041033.

[4] Wu W., Yang J., Zhang J., Huang J., Chen D., WANG H., Ultra-high resolution filter and optical field modulator based on a surface plasmon polariton, Optics Letters 41(10), 2016, pp. 2310-2313, DOI: 10.1364/OL.41.002310.

[5] Wang H., Yang J., Zhang J., Huang J., Wu W., Chen D., XIao G., Tunable band-stop plasmonic waveguide filter with symmetrical multiple-teeth-shaped structure, Optics Letters 41(6), 2016, pp. 1233-1236, DOI: 10.1364/OL.41.001233.

[6] Zavvari M., Taleb Hesami Azar M., Arashmehr A., Tunable band-stop plasmonic filter based on square ring resonators in a metal-insulator-metal structure, Journal of Modern Optics 64(20), 2017, pp. 2221-2227, DOI: 10.1080/09500340.2017.1349195. 
[7] SAHU P.P., Theoretical investigation of all optical switch based on compact surface plasmonic two mode interference coupler, Journal of Lightwave Technology 34(4), 2015, pp. 1300-1305.

[8] Wang G., Lu H., Liu X., Gong Y., Numerical investigation of an all-optical switch in a graded nonlinear plasmonic grating, Nanotechnology 23(44), 2012, article ID 444009, DOI: 10.1088/0957 $-4484 / 23 / 44 / 444009$.

[9] Nozhat N., Granpayeh N., All-optical nonlinear plasmonic ring resonator switches, Journal of Modern Optics 61(20), 2014, pp. 1690-1695, DOI: 10.1080/09500340.2014.951008.

[10] Kuang D., Dong L., CaO Y., Tuning of plasmonic nanofocusing with non-linear metallic helical nanocone, Plasmonics 12, 2017, pp. 685-690, DOI: 10.1007/s11468-016-0314-2.

[11] Niv L., XiAng Y., Luo W., CAI W., Qi J., Zhang X., Xu J., Nanofocusing of the free-space optical energy with plasmonic Tamm states, Scientific Reports 6, 2016, article ID 39125, DOI: 10.1038/srep39125.

[12] Chen X., Lindquist N.C., Klemme D.J., Nagpal P., Norris D.J., Oh S.-H., Split-wedge antennas with sub-5 nm gaps for plasmonic nanofocusing, Nano Letters 16(12), 2016, pp. 7849-7856, DOI: 10.1021/acs.nanolett.6b04113.

[13] Wu D., Liu Y., Yu L., Yu Z., Chen L., Li R., Ma R., Liu C., Zhang J., Ye H., Plasmonic metamaterial for electromagnetically induced transparency analogue and ultra-high figure of merit sensor, Scientific Reports 7, 2017, article ID 45210, DOI: 10.1038/srep45210.

[14] Zhao C., Song X., Jiao R., Duan G., Wang L., Yu L., Tunable electromagnetically induced transparency in plasmonic system and its application in nanosensor and spectral splitting, IEEE Photonics Journal 7(6), 2015, pp. 1-8, DOI: 10.1109/JPHOT.2015.2492550.

[15] Wei Z., Li X., Zhong N., Tan X., Zhang X., Liu H., Meng H., Liang R., Analogue electromagnetically induced transparency based on low-loss metamaterial and its application in nanosensor and slow -light device, Plasmonics 12, 2017, pp. 641-647, DOI: 10.1007/s11468-016-0309-z.

[16] CHEN C., SHENG Y., Jun W., Computed a multiple band metamaterial absorber and its application based on the figure of merit value, Optics Communications 406, 2018, pp. 145-150, DOI: 10.1016/j.optcom. 2017.06.009.

[17] Yang W.H., Zhang C., Sun S., Jing J., Song Q., XIAo S., Dark plasmonic mode based perfect absorption and refractive index sensing, Nanoscale 9(26), 2017, pp. 8907-8912, DOI: 10.1039/C7NR02768K.

[18] Wu D., Li R., Liu Y., Yu Z., Yu L., Chen L., Liu C., MA R., Ye H., Ultra-narrow band perfect absorber and its application as plasmonic sensor in the visible region, Nanoscale Research Letters $\mathbf{1 2}$, 2017, article ID 427, DOI: 10.1186/s11671-017-2203-9.

[19] Boller K.-J., ImAmoĞLu A., HarRis S.E., Observation of electromagnetically induced transparency, Physical Review Letters 66(20), 1991, pp. 2593-2596, DOI: 10.1103/PhysRevLett.66.2593.

[20] Chen J., Gan F., Wang Y., Li G., Plasmonic sensing and modulation based on Fano resonances, Advanced Optical Materials 6(9), 2018, article ID 1701152, DOI: 10.1002/adom.201701152.

[21] Tonouchi M., Cutting-edge terahertz technology, Nature Photonics 1, 2007, pp. 97-105, DOI: $10.1038 /$ nphoton.2007.3.

[22] Ferguson B., Zhang X.C., Materials for terahertz science and technology, Nature Materials 1, 2002, pp. 26-33, DOI: $10.1038 /$ nmat708.

[23] Chernomyrdin N.V., Schadko A.O., Lebedev S.P., Tolstoguzov V.L., Kurlov V.N., Reshetov I.V., Spektor I.E., Skorobogatiy M., Yurchenko S.O., Zaytsev K.I., Solid immersion terahertz imaging with sub-wavelength resolution, Applied Physics Letters 110(22), 2017, article ID 221109, DOI: $10.1063 / 1.4984952$.

[24] Smith R., Jooshesh A., Zhang J., Darcie T., Photoconductive generation and detection of THz-bandwidth pulses using near-field coupling to a free-space metallic slit waveguide, Optics Express 25(22), 2017, pp. 26492-26499, DOI: 10.1364/OE.25.026492.

[25] Gao F., Zhu Z., Xu W., Zhang J., Guo C., Liu K., Yuan X., Qin S., Broadband wave absorption in single-layered and nonstructured graphene based on far-field interaction effect, Optics Express 25(9), 2017, pp. 9579-9586, DOI: 10.1364/OE.25.009579. 
[26] Hu B., WANG Q.J., Zhang Y., Broadly tunable one-way terahertz plasmonic waveguide based on nonreciprocal surface magneto plasmons, Optics Letters 37(11), 2012, pp. 1895-1897, DOI: 10.1364 / OL.37.001895.

[27] TaO J., Hu B., He X.Y., Wang Q.J., Tunable subwavelength terahertz plasmonic stub waveguide filters, IEEE Transactions on Nanotechnology 12(6), 2013, pp. 1191-1197, DOI: 10.1109/TNANO. 2013.2285127.

[28] Hu B., Tao J., Zhang Y., Wang Q.J., Magneto-plasmonics in graphene-dielectric sandwich, Optics Express 22(18), 2014, pp. 21727-21738, DOI: 10.1364/OE.22.021727.

[29] Li W., Kuang D., Fan F., Chang S., Lin L., Subwavelength B-shaped metallic hole array terahertz filter with InSb bar as thermally tunable structure, Applied Optics 51(29), 2012, pp. 7098-7102, DOI: 10.1364/AO.51.007098.

[30] Girard M., SкоRobogatiy M., Integrated terahertz multiparameter sensors using fiber/frequency selective surface couplers, Journal of Optics 16(9), 2014, article ID 094007, DOI: 10.1088/2040-8978/ 16/9/094007.

[31] SÁncheZ-Gil J.A., Rivas J.G., Thermal switching of the scattering coefficients of terahertz surface plasmon polaritons impinging on a finite array of subwavelength grooves on semiconductor surfaces, Physical Review B 73(20), 2006, article ID 205410, DOI: 10.1103/PhysRevB.73.205410.

[32] OszWalLdowski M., ZimPel M., Temperature dependence of intrinsic carrier concentration and density of states effective mass of heavy holes in InSb, Journal of Physics and Chemistry of Solids 49(10), 1988, pp. 1179-1185, DOI: 10.1016/0022-3697(88)90173-4.

[33] Howells S.C., Schlie L.A., Transient terahertz reflection spectroscopy of undoped InSb from 0.1 to $1.1 \mathrm{THz}$, Applied Physics Letters 69(4), 1996, pp. 550-552, DOI: 10.1063/1.117783.

[34] DaI X., XIANG Y., Wen S., He H., Thermally tunable and omnidirectional terahertz photonic bandgap in the one-dimensional photonic crystals containing semiconductor InSb, Journal of Applied Physics 109(5), 2011, article ID 053104, DOI: 10.1063/1.3549834.

[35] Manolatou C., Khan M.J., Fan S., Villeneuve P.R., Haus H.A., Joannopoulos J.D., Coupling of modes analysis of resonant channel add-drop filters, IEEE Journal of Quantum Electronics 35(9), 1999, pp. 1322-1331, DOI: 10.1109/3.784592.

[36] Xiao Y.-F., Li M., Liu Y.-C., Li Y., Sun X., Gong Q., Asymmetric Fano resonance analysis in indirectly coupled microresonators, Physical Review A 82(6), 2010, article ID 065804, DOI: $10.1103 /$ PhysRevA.82.065804.

[37] Miyazaki H.T., KuroKawa Y., Squeezing visible light waves into a 3-nm-thick and 55-nm-long plasmon cavity, Physical Review Letters 96(9), 2006, article ID 097401, DOI: 10.1103/PhysRevLett.96.097401.

Received September 3, 2018 in revised form October 16, 2018 